\title{
Estrategias de solución de problemas aplicadas por ajedrecistas de diferente nivel
}

\section{Problem solving strategies in chess players of different levels}

\author{
Luis Lázaro Aguiero Jiménez \\ Natividad C. Lainé Oquendo** \\ Universidad de la Habana, Cuba \\ Recibido: 20 de febrero de 2016 \\ Revisado: 30 de marzo de 2016 \\ Aceptado: 3 de mayo de 2016
}

\section{Resumen}

En el presente trabajo se estudiaron las diferentes estrategias de solución de problemas aplicadas por ajedrecistas de distinto nivel, y así desarrollar programas más integradores que permitan perfeccionar el entrenamiento de ajedrecistas de todas las edades. Por esto, lo estudiado en la presente investigación sirve como guía para la captación de jóvenes talentos que deseen integrar las escuelas deportivas que posee el país; y desde el punto de vista psicológico, lo más relevante es la manera en que los ajedrecistas dan soluciones a problemas que se les presentan en la vida cotidiana a partir del aprendizaje que reciben en el juego de ajedrez. Se tuvo como objetivo identificar las estrategias de solución de problemas en ajedrecistas de diferentes niveles, del cual se derivan los objetivos específicos de: determinar las estrategias que utilizan los ajedrecistas de la muestra en tareas de pensamiento lógico, definir las estrategias que utilizan los ajedrecistas de la muestra en la solución de partidas de ajedrez y el determinar si los ajedrecistas con mayor experiencia practicando este deporte utilizan estrategias de solución de problemas más eficaces; de estos objetivos específicos se extrajeron las varia- 
bles: resolución de problemas, pensamiento, pensamiento lógico, estrategia, y eficacia, las cuales fueron medidas con el test de ajedrez propuesto por Przewoznik y Soszynski (2004) y por el test de pensamiento lógico de Tobin y Capie (1981) La muestra estuvo conformada por siete sujetos y los resultados de ambos test fueron procesadas con el programa Microsoft Excel. Se logró constatar en los resultados que en el test de ajedrez también se aplican los cinco esquemas propuestos en el test de pensamiento lógico (proporcionalidad, control de variables, probabilidad, correlación y operaciones combinatorias), y aquellos jugadores de más experiencia fueron más eficaces en la solución de las posiciones de ajedrez, mientras que en el test de Tobin y Capie los resultados manifestaron una gran paridad en cuanto a las estrategias realizadas.

Palabras clave: resolución de problemas, pensamiento, estrategia, pensamiento lógico, eficacia.

\section{Abstract}

We studied the different problem solving strategies applied by chess players of different levels, with the goal of creating more intregrative programmes that are better at perfecting the training of chess players of all ages. Hence, this research might guide the selection of young talented players in sports schools in our country, and from the psychological point of view, the most relevant issue is how chess players solve problems in daily life using what they have learned through chess. The main goal was to identify problem solving strategies in chess players of different levels, and the specific goals were to determine the strategies used by chess players in logical thinking tasks, to define the strategies used by the sample in the solution of chess games, and to determine whether more experienced players use better strategies. The variables were: problem solving, logical thinking, thinking, strategy, and efficacy, measured with the Chess Test proposed by Przewoznik y Soszynski (2004) and the logical thinking test by Tobin \& Capie (1981). The sample consisted of eight participants, and data were processed with Excel. We found that the five domains proposed in the logical thinking test (proportionality, variable control, probability, correlation, and combinatorial operations) are also present in the Chess Test, and that more experienced players had higher efficacy at solving chess positions. The Tobin and Capie test showed large similarities in terms of strategies.

Keywords: Problem solving, Thinking, Strategy, Logical Thinking, Efficacy

\section{Introducción}

Es importante señalar que siempre ha llamado la atención el hecho de que los ajedrecistas tengan un buen desempeño docente o en la vida cotidiana, debido a una excelente memoria, concentración, rapidez en la toma de decisiones y descubrimiento de ideas esenciales que conllevan a un gran resumen de cualquier contenido.

Es vital entonces realizar preguntas que deberán ser respondidas y que cimentan la base de ideas que van en busca de avanzar en los estudios que relacionan a la psicología con el ajedrez, entre ellas: ¿cuáles estrategias aplican los practicantes de ajedrez de diferentes niveles en tareas de pensamiento lógico?, ¿cuáles estrategias aplican los practicantes de ajedrez de diferentes niveles en la solución de partidas de ajedrez?, ¿los ajedrecistas que llevan más tiempo practicando ajedrez utilizan estrategias de solución de problemas más eficaces?

De lo anterior queda planteada la gran necesidad e importancia del estudio, ya que en primer lugar es sabido que el ajedrez es uno de los deportes que en los últimos años ha traído grandes momentos al deporte cubano, sin embargo, carece muchas veces de un trabajo psicológico científico capaz de ayudar y potenciar la fortaleza de este 
deporte en Cuba. Como segundo elemento es el hecho de la relevancia nacional que pudiera ofrecer esta investigación, puesto que no hay trabajos suficientes en el país que logren de manera científica consolidar estos nexos entre la resolución de problemas y el ajedrez. Como un tercer elemento está la importancia de verificar cómo la práctica del ajedrez se manifiesta en la eficacia de las estrategias para la solución de problemas, ya que de esa manera se le daría una confirmación científica al tema de la masificación del ajedrez para el desarrollo personal de cada ser humano que se encuentre inclinado a practicarlo.

Desde el punto de vista científico es vital comprender cómo genera desarrollo del pensamiento el jugar ajedrez a partir de la tarea constante de la solución de problemas, que encierra dentro de sí este deporte, a partir de una ordenación y transformación cada vez que los ajedrecistas se encuentran en una posición de una partida; es por ello que el ajedrez cumple los requisitos necesarios para abordar al pensamiento desde el enfoque de procesamiento de la información.

Es por lo antes expuesto que el presente trabajo pretende como objetivo general identificar las estrategias de solución de problemas en ajedrecistas de diferentes niveles, del cual se desprenden objetivos más específicos como el determinar las estrategias que utilizan los ajedrecistas de la muestra en tareas de pensamiento lógico, definir las estrategias que utilizan los ajedrecistas de la muestra en la solución de partidas de ajedrez y determinar si los ajedrecistas con mayor experiencia practicando este deporte utilizan estrategias de solución de problemas más eficaces; de las pretensiones que se quieren con el presente trabajo se deduce que la combinación psicología y ajedrez es de suma importancia para aquellos jugadores que aspiren a convertirse en excelentes jugadores profesionales. Por ello cada jugador debe tener sus propios métodos de autodisciplina, régimen de torneo, cultivo de desempeño y un óptimo pensamiento que se traduce no solo en las situaciones técnicas de cada partida, sino también a la hora de organizarlos con el fin de saber qué se quiere en cada momento competitivo.
En tal sentido, para ese óptimo pensamiento es vital el establecimiento de metas y objetivos que sirvan para el desarrollo de un jugador de ajedrez; por mencionar algunos en su vínculo con el ajedrez citar: el de ser Gran Maestro (el cual es el objetivo principal de la carrera), analizar y dejar anotados dichos análisis, el consultar la literatura ajedrecística y crear un catálogo de posiciones más importantes de partidas y posiciones clásicas.

Consecuentemente, como otro pilar de orden psicológico en el ajedrez, es el desarrollo de una filosofía minuciosa de pensamiento positivo, afirmativo y realista.

Se puede exponer entonces que algunos de los logros del ajedrez radican en la memoria visual, el poder combinatorio, la velocidad para calcular, el poder de concentración y el pensamiento lógico.

Por ello se debe hacer referencia a la reunión de la Comisión de Ajedrez en la Educación de la Federación Internacional de Ajedrez (FIDE) de 1984, donde se mencionan los beneficios del ajedrez en el desarrollo intelectual de hombres y mujeres tales como: desarrollo de la memoria, incremento de la creatividad, enriquecimiento cultural y desarrollo mental. Se debe añadir además que el ajedrez beneficia socialmente al niño y le da gran madurez de carácter.

También se puede hacer alusión a la investigación titulada: Valor educativo del ajedrez, en donde se explica que en el transcurso de una partida de ajedrez, así como en la resolución de problemas, discurre una forma de pensamiento organizado que se asemeja al discurso científico. En este documento se manifiesta que en el acto del desarrollo de una partida, el ajedrecista observa, compara, clasifica, organiza ideas, hipotetiza aquello que investiga, analiza, ensaya, sintetiza, toma decisiones y las ejecuta. De ahí que se señale que la práctica sistemática del ajedrez comparte con la escuela el propósito de desarrollo del pensamiento, la enseñanza de valores y el facilitar hábitos virtuosos del carácter. Además, estimula la expresión numérica y verbal, la autoestima y la creatividad. En dicho texto se cita al psicólogo Adrian de Groot, quien en 1960 realizó un estudio en la Unión Soviética entre dos grupos 
de niños y jóvenes de diferentes edades pero con características comunes. A un grupo le enseñaron el juego y al otro no, después de un año observó que al grupo que se le vinculó al juego-ciencia tuvo un adelanto extraordinario en el uso racional de la lógica, la capacidad de concentración, el desarrollo de la memoria, la disciplina mental y la madurez emocional.

Por otro lado, el programa de ajedrez de las escuelas de la ciudad de Nueva York fue fundado en 1986 por Faneuil Adams, Jr. y Bruce Pandolfini. El programa envía a un instructor experimentado a las escuelas para establecer un programa de ajedrez que ayuda a los estudiantes a mejorar sus notas, especialmente en las materias de inglés y matemáticas; fortalece el sentido de trabajo en equipo a la vez que realza las habilidades de la persona; hace que el niño dé valor a trabajar arduamente, concentrarse y empeñarse, se dé cuenta que es responsable de sus propios actos y les enseña a dar lo mejor de sí, proveyéndose de victorias o aceptando con gracia las derrotas, es un foro intelectual, competitivo, puede llegar a ser una actividad en la cual se enfocan las energías de una forma aceptable, mejora el nivel de asistencia en una forma que permita a las niñas competir con los niños sin sentirse intimidadas, ayuda a los niños a establecer amistades y proporciona un escenario agradable y seguro que permite a los estudiantes y maestros reunirse y discutir entre ellos.

Durante mucho tiempo los problemas de ajedrez han servido para aplicárselos a jugadores profesionales de dicho juego, con el objetivo de desarrollar la velocidad de cálculo, el poder combinatorio, la valoración, la táctica y la estrategia por solo citar algunos de los elementos de este complejo pero fascinante mundo.

Desde el punto de vista práctico, vale la pena citar que en Cuba los problemas de ajedrez han sido aplicados a la preselección nacional masculina y femenina, a los jugadores de las preselecciones provinciales y atletas de excelente desempeño internacional.

En el caso de los ejercicios del test de Przewoznik y Soszynsky se aplicaron a diferentes jugadores de Polonia. Durante algún tiempo los resultados se publicaron en varias revistas y libros que al final se recopilaron y se les dio un carácter más profundo y científico.

En cuanto al Test de Tobin y Capie (TOLT, 1981) se compone de los siguientes esquemas de razonamiento lógico: proporcionalidad (PP, preguntas 1 y 2 ), control de variables ( $\mathrm{CV}$, preguntas 3 y 4 ), probabilidad (PR, preguntas 5 y 6 ) correlación (CR, preguntas 7 y 8 ) y operaciones combinatorias (OP, preguntas 9 y 10).

\section{Método}

La presente es una investigación de carácter cualitativa-cuantitativa, con un diseño no experimental en donde se utilizó una perspectiva transeccional-descriptiva, que se apoyará en un enfoque de estudio de casos.

\section{Participantes}

Se ha llegado a tener siete sujetos de muestra que van a ser descritos de acuerdo a los parámetros de la siguiente tabla:

Tabla 1.

Sujetos de muestra.

\begin{tabular}{ccccc}
\hline Sujeto & Edad & Título en ajedrez & Años de experiencia & Mejor resultado \\
\hline MRP & 13 & - & 2 & $2 .{ }^{\circ}$ lugar provincial 12-14 \\
\hline RAA & 12 & MF & 4 & Campeón panamericano sub-12 \\
\hline JRR & 15 & - & 11 & $2 .{ }^{\circ}$ lugar provincial juvenil 2014 \\
\hline YML & 32 & GM & 22 & Oro olímpico 2015 \\
\hline RMG & 13 & - & 5 & Bronce centroamericano sub-12 \\
\hline VOM & 14 & - & 4 & $2 .{ }^{\circ}$ lugar nacional 10-11 \\
\hline RGP & 22 & $M I$ & 14 & $2 .{ }^{\circ}$ lugar empatado en el Capablanca in Memorian \\
\hline
\end{tabular}

Fuente: megadatabase 2014 y página oficial de la FIDE. 
En la tabla anterior se puede apreciar que la muestra escogida tiene un promedio de edad de 17.2 años, lo cual hace precisamente que sea una población joven, sin embargo, el $57 \%$ de la muestra posee una experiencia igual o superior a los 5 años de práctica del ajedrez, y aquellos que están por debajo de este rango han demostrado en sus resultados que son poseedores de un gran talento a pesar de su corta experiencia.

\section{Técnicas e instrumentos}

Test de razonamiento lógico de Tobin y Capie (1981): este test de razonamiento lógico consiste en un cuestionario de diez tareas para realizar con papel y lápiz, dos por cada uno de los siguientes esquemas de razonamiento: proporcionalidad (PP, preguntas 1 y 2 ), control de variables ( $\mathrm{CV}$, preguntas 3 y 4), probabilidad (PR, preguntas 5 y 6), correlación (CR, preguntas 7 y 8 ) y operaciones combinatorias (OP, preguntas 9 y 10). Las ocho primeras cuestiones constan de dos niveles, respuesta-explicación, diseñadas con un formato de opción múltiple tanto en lo que se refiere a la respuesta como a su correspondiente justificación. Tanto las respuestas como las explicaciones sugeridas como posibles alternativas, corresponden a algunos de los errores sistemáticos más frecuentes en los que suele incurrirse en la resolución de este tipo de problemas (Garnett y Tobin, 1984; Garnett, Tobin y Swingler, 1985; Acebedo y Romero, 1991). Por el contrario, las dos últimas preguntas, referentes a combinaciones y permutaciones son de respuesta abierta semiestructura$\mathrm{da}$, donde los sujetos disponen de un total de 38 minutos para la realización de la prueba.

Test de ajedrez de Przewoznik y Soszynski (2004): se presentarán dos de las posiciones propuestas por los autores de la prueba y se tendrá 30 minutos para montar cada una en el tablero y luego encontrar la mejor jugada en conjunto con algunos planes y variantes. Es muy importante recordar que esta tarea no es solamente para que se escoja un movimiento en cada posición, sino que se deben expresar los pensamientos escritos y en voz alta durante los cálculos de variantes. Los pensamientos pueden ser grabados en una cinta de audio. Es vital que todo lo que venga a la mente durante la elección de un movimiento sea dicho en voz alta; es importante destacar también que todos los pensamientos sobre la posición son importantes. Tiene que pensar que la prueba es un juego normal de un torneo, mas, diferentemente de lo que ocurre en un torneo, hay que hablar todo lo que se ve, verifica o planea.

Para hallar la solución se especifica en cada posición a quién le corresponde jugar, si a las blancas o a las negras. Si la situación es realmente posicional o táctica y si se puede vencer, empatar o perder, eso debe ser descubierto por el sujeto estudiado.

Figura 1. Blancas: Lutikov. Negras: Taimanov.

Posición 1

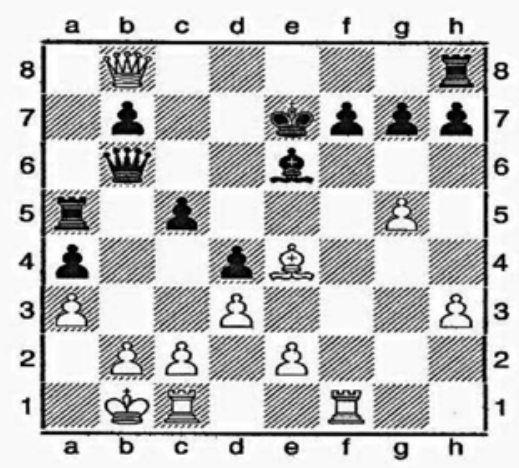

Respuesta: $1 . \mathrm{Tf7}$

Fuente: USSR, 1969.

Figura 2. Blancas: Konstantinopolsky. Negras: Aronin.

Posición 2

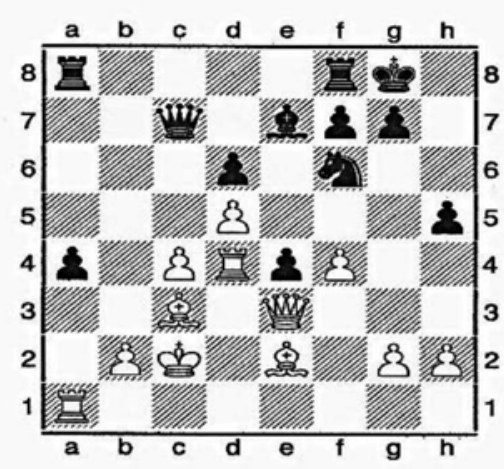

Respuesta:1.g4

Fuente: Moscú, 1950. 


\section{Observación}

Brinda información acerca del lenguaje corporal, tan importante en el ajedrez puesto que permite descifrar estados como la ansiedad o la falta de concentración durante los problemas del llamado deporte-ciencia.

\section{Entrevista}

En este caso se utilizaría una entrevista no estructurada con el objetivo de obtener información adicional durante la resolución de problemas, y de ver algunas cuestiones que no queden claras en cuanto a la forma de procesamiento en que los sujetos de estudio realizan las pruebas.

\section{Análisis de documentos}

Durante el presente trabajo se utilizará este método con el objetivo de caracterizar a los ajedrecistas de la muestra, fundamentalmente se revisará la lista ELO (rating de los ajedrecistas) con el fin de ubicar el nivel que poseen, integrado por supuesto con los resultados obtenidos en diferentes competencias.

\section{Introspección}

Con el pensamiento en voz alta los sujetos de la muestra serán conscientes de lo que están diciendo, y de esa manera buscan y expresan dentro de sí y hacia el medio las diferentes estrategias solucionadoras de problemas que se les presenten.

Aunque todo lo antes expuesto se analiza por separado, al final se hace una integración de dichos resultados que permite evaluar si el pensamiento formal ajedrecístico de los individuos fue elevado o no.

\section{Resultados}

En sentido general, se puede hablar de atletas que muestran buena concentración y disciplina durante las técnicas utilizadas en las sesiones. En el caso del test de pensamiento lógico, la mayoría de la muestra evidenció un pensamiento formal bajo, aunque dos sujetos se destacaron al tener un pensamiento lógico elevado; sin embargo, todos fallaron en la utilización del esquema de proporcionalidad, representado en las preguntas 1 y 2 del test anteriormente mencionado, tal y como se manifiestan en los promedios de la tabla 2:

Tabla 2.

Promedios.

\begin{tabular}{cc}
\hline Esquema & Promedio \\
\hline PP & 0 \\
\hline CV & 0,64285714 \\
\hline PB & 0,35714286 \\
\hline CR & 0,5 \\
\hline OP & 0,71428571 \\
\hline
\end{tabular}

Fuente: resultados del test de pensamiento lógico.

En la tabla anterior se muestran también que los sujetos estudiados tienen la mejor calificación en el esquema de operaciones combinatorias, lo cual demuestra las permutaciones que deben hacerse en las situaciones propuestas en el test, y que es manifestación también de la práctica que tienen estos atletas en dicha estrategia, pues en ajedrez esto se debe hacer durante el cálculo de variantes.

El esquema de control de variables tuvo buenos resultados, al igual que el de correlación como manifestación de la conjunción de las diferentes variables existentes para dar solución a los problemas.

Sin embargo, el esquema de probabilidad tuvo también resultados negativos, lo cual denota la dificultad de manejar de manera espacial en la mente los elementos matemáticos necesarios para dar respuesta a ambos ítems. Lo expresado en los párrafos anteriores demuestra las deficiencias existentes en cuanto a la elección de las respuestas, debido a que no se desecharon aquellas variables o alternativas que no eran verdaderas en función de lograr el mayor éxito posible en la prueba; esto, por supuesto, trae consigo un repercusión directa en las estrategias aplicadas por cada uno de los sujetos, ya que evidenció dificultades en la analogía de las situaciones y el control 
de variables enmarcado en la valoración que se debe hacer de cualquier problema.

Por otro lado, se tiene que en el test de ajedrez, las estrategias se enfocaron desde el punto de vista técnico, en la explotación de debilidades, el ataque al rey, destrucción del refugio de peones y la iniciativa mediante la mejor ubicación de cada una de las piezas; sin embargo, se manifestaron problemas en la valoración de la posición como paso inicial clave para lograr la mayor efectividad en cualquier situación problémica, incluso aquellos sujetos que la realizaron no captaron la esencia de la posición y por tanto su valoración no fue tampoco efectiva al $100 \%$; el cálculo de variantes también fue un elemento de deficiencias puesto que solo las líneas forzadas eran las que llegaban o sobrepasaban la barrera de las cinco jugadas, cuando habían varias alternativas ese cálculo llegaba con fortaleza solo a los tres primeros movimientos.

Los datos cuantitativos ofrecidos en el análisis de la técnica de ajedrez, proporcionan evidencia de que también hubo dificultades en la uniformidad de la jugada escogida, pues en sentido general se muestran valores bajos que son interpretados como la poca proporción de tiempo usado para esa variante final; sin embargo, se muestra una mejoría cuando se incluye el análisis de jugadas superiores con respecto a las inferiores para dar una mejor economía de pensamiento.

Los siguientes datos de la tabla 3 muestran los resultados alcanzados por los sujetos de la muestra en las dos posiciones presentadas:

Tabla 3.

Resultados alcanzados.

\begin{tabular}{lccccccc}
\hline Sujeto & A & N & E & Pmax & Dmax & Vf & T(m) \\
\hline \multirow{2}{*}{ MRP } & Pos1:6 & Pos1:7 & Pos1:19 & Pos1:2 & Pos1:6 & Pos1:De5 & Pos1:18 \\
\cline { 2 - 7 } RAA & Pos2:5 & Pos2:7 & Pos2:19 & Pos2:3 & Pos2:5 & Pos2:Te4 & Pos2:21 \\
\cline { 2 - 7 } & Pos1:4 & Pos1:12 & Pos1:38 & Pos1:3 & Pos1:5 & Pos1:De5 & Pos1:22 \\
\hline \multirow{2}{*}{ JRR } & Pos2:1 & Pos2:5 & Pos2:5 & Pos2:1 & Pos2:5 & Pos2:Te4 & Pos2:14 \\
\cline { 2 - 7 } & Pos2:3 & Pos1:12 & Pos1:28 & Pos1:2 & Pos1:5 & Pos1:Tf7 & Pos1:25 \\
\hline \multirow{2}{*}{ YML } & Pos1:4 & Pos1:12 & Pos1:26 & Pos1:5 & Pos1:6 & Pos1:Tf7 & Pos1:25 \\
\cline { 2 - 7 } RMG & Pos2:2 & Pos2:8 & Pos2:9 & Pos:1 & Pos2:7 & Pos2:f5 & Pos2:25 \\
\cline { 2 - 7 } Vom & Pos1:3 & Pos1:7 & Pos1:10 & Pos1:5 & Pos1:5 & Pos1:De5 & Pos1:25 \\
\cline { 2 - 7 } & Pos2:1 & Pos2:9 & Pos2:9 & Pos2:9 & Pos2:4 & Pos2:Te4 & Pos2:25 \\
\hline \multirow{2}{*}{ RGP } & Pos2:2 & Pos1:12 & Pos1:20 & Pos1:6 & Pos1:5 & Pos1:Db7 & Pos1:23 \\
\cline { 2 - 7 } & Pos1:4 & Pos1:11 & Pos1:25 & Pos1:3 & Pos1:5 & Pos1:De5 & Pos1:25 \\
\hline
\end{tabular}

Fuente: resultados del test de ajedrez.

(A) Conjunto de movimientos candidatos: conjunto de alternativas durante el proceso de toma de decisiones.

(N) Número total de soluciones sucesivas propuestas: chequeo y cálculo de un gran número de variantes en una posición dada.

(e) Suma de clasificaciones: cada letra en la fórmula de propuestas de soluciones sucesivas puede ser atribuida a una clasificación apropiada. De esa manera cuanto más fuerte el movimiento considerado, menor la calificación dada.

(Pmax) Número de soluciones reexaminadas: son todas las soluciones evaluadas más de una vez.

(Dmax) Número máximo de jugadas en las variantes de las blancas y las negras: cantidad de jugadas del blanco y del negro con el fin de prever y descubrir los eventos de las posiciones.

(Vf) Variante final: respuesta de la posición en cuestión.

(T) Tiempo para resolver los ejercicios: tiempo en que el individuo se tarda resolviendo cada posición. 
Durante la introspección se logra constatar la rigidez del pensamiento en función de hallar variantes forzadas y lineales, esto hace que escapen las mejores jugadas y planes, y tenga como consecuencia directa el no encontrar las mejores soluciones en la posición; además se evidenció que durante el proceso de toma de decisiones, el pensamiento se tornaba redundante y esto hacía que se perdiera tiempo y organización para elegir una alternativa.

A la hora de diseccionar la muestra estudiada en aquellos que tienen mayor experiencia, se nota que los poseedores de más de diez años de experiencia de práctica en ajedrez tienen un resultado ligeramente superior en esas situaciones problémicas, sin embargo, en el test de pensamiento lógico se mostraron cercanos al promedio a excepción del sujeto JRR, quien tuvo una calificación excelente; este sujeto en los problemas de ajedrez tuvo además $50 \%$ de efectividad al tener un problema correcto y el otro no, que lo ubica en el atleta más destacado, lo contrario pasa con el sujeto VOM quien se destacó en el test de pensamiento lógico, pero fue poco efectivo en los problemas de ajedrez.

Lo expuesto hasta el momento al hablar de la generalidad de la muestra, hace deducir que las estrategias de pensamiento aplicadas en los problemas muestran una gran paridad, es precisamente la experiencia, que conlleva horas de entrenamiento, partidas de torneo y posiciones vistas, unido a un primer análisis general de la situación problémica lo que hace que las jugadas en el test de ajedrez sean más fuertes y flexibles en los jugadores que llevan más de diez años practicando este deporte, y que logra marcar esa diferencia en cuanto a la exactitud de la toma de decisiones.

Por ello sería propicio reafirmar que ese estudio de varias horas, entrenamiento diario, ejercitación de posiciones de ajedrez y el jugar de manera frecuente, son la vía para potenciar las virtudes de solución de problemas y toma de decisiones, y de esa manera erradicar las dificultades mostradas a lo largo del presente trabajo por cada uno de los sujetos, y hacer más profundo y complejo el pensamiento lógico en cada situación problémica que se presente a lo largo no solo del ajedrez sino también de la vida.

\section{Conclusiones}

1. En el test de pensamiento lógico se presentaron los esquemas y estrategias de proporcionalidad, control de variables, probabilidad, correlación y operaciones combinatorias; mostrando paridad en los resultados en la mayoría de los sujetos, ya que fueron muy parecidas las formas en que estas estrategias se pusieron en práctica por cada uno de los ajedrecistas estudiados, para dar respuesta a cada una de las situaciones presentadas en el test.

2. En el test de ajedrez se aplicaron estas mismas estrategias de pensamiento lógico, y desde el punto de vista ajedrecístico, los elementos tácticos más usados fueron el ataque al rey, la iniciativa y la destrucción de la defensa que respondieron a una estrategia general de lograr ventaja en cada posición a partir de una valoración que responde a los factores más importantes en cada situación problémica.

3. La eficacia de las soluciones de los problemas de ajedrez fue mayor en los atletas de más experiencia, que se orientaron de manera más eficiente al tener en cuenta jugadas candidatas más exactas que respondían a las demandas de cada una de las posiciones del juego. En este sentido se les hizo más fácil el cálculo de variantes y por supuesto también tuvieron una mayor economía de tiempo.

4. La valoración de las situaciones problémicas fue la mayor debilidad presentada en los sujetos, pues en sentido general se obviaron elementos posicionales que eran indispensables para elaborar estrategias y planes que sirvieran para la mejor orientación de la posición; además de que estos elementos también eran importantes para la visualización de cada una de las posiciones resultantes luego de los cálculos de variantes. 


\section{Referencias}

Alexeiev, N. G. (s.f.). El ajedrez en el desarrollo del pensamiento (digital). Edami.

Colectivo de autores. (2003). Ajedrez Integral. Tomo 1. La Habana: Editorial Deportes.

Colectivo de autores. (2005). Ajedrez Integral. Tomo 2. La Habana: Editorial Deportes.

Colectivo de autores. (2005). Estrategia. La Habana: Convekta.

Colectivo de autores. (2006). Psicología del desarrollo del escolar. Selección de lecturas. La Habana: Editorial Félix Varela.

Colegio Alcántara. (2010). Valor educativo del ajedrez. Recuperado de www.alcantara/penalolen/noticias/archivoajedrez/ajedrez-valoreducativo

Cruz, L. (1991). Psicología del desarrollo. España: Editorial Alianza.

Domínguez, L. (2005). Psicología del desarrollo, problemas, principios y categorías. La Habana: Facultad de Psicología.

Dvoretsky, M. (2002). Secretos del entrenamiento en ajedrez. España: Editorial Nerán.

Ecured. (1993). Lógica y procedimientos lógicos del pensamiento. Recuperado de www. ecured.cu/index.php?title=Pensamiento_ lógico\&oldid $=943356$

Eficacia y eficiencia. (2015). El reto de actuar con equilibrio. Recuperado de http://contaduriapublica.org.mx/eficacia-y-eficiencia-el-retode-actuar-con-equilibrio/.

González, A., Fuentes, M., de La Morena, M., y Barajas, C. (1995). Psicología del desarrollo: teorías y prácticas. España: Editorial Aljibe.
Kasparov, G. (2007). Cómo la vida imita al ajedrez. México: Editorial Grijalbo.

Manzano, M. (2013). Temas de psicología cognitiva 1. Selección de lecturas. La Habana: Editorial Félix Varela.

Manzano, M. (2013). Temas de psicología cognitiva 2. Selección de lecturas. La Habana: Editorial Félix Varela.

Pérez, Y. (2007). Programa de orientación psicológica para estimular el autocontrol emocional en ajedrecistas de alto rendimiento. Tutores: doctor Luis Felipe Herrera y Magda Mesa Anoceto. Trabajo de doctorado. La Habana, Cuba: Universidad de la Cultura Física y el Deporte Manuel Fajardo.

Przewoznik, J. y Soszynski, M. (2004). Cómo pensar en ajedrez. Río de Janeiro: Editorial Ciencia Moderna.

Rowson, J. (2000). Los siete pecados capitales del ajedrez. España: Editorial La Casa del Ajedrez.

Sampieri, R., Collado, C., y Lucio, P. (2006). Metodología de la investigación. México: Editorial McGraw-Hill.

Psicopediahoy. (2012). Niveles de pensamiento en estudiantes de psicología de una universidad pública de la región caribe-colombiana. Recuperado de www.psicopediahoy.com/niveles-de-pensamiento-estudiantes-universidadpublica/

Slideshare. (1996). Teorías de Piaget. Recuperado de www.es.slideshare.net/guestacef4d/3teora-de-piaget

Vega, J. L. y Bueno, B. (1996). Desarrollo adulto y envejecimiento. España: Editorial Síntesis.

Vega, M. (1994). Introducción a la psicología cognitiva. Tomo 2. Madrid: Editorial Alianza. 